\title{
Quantum antenna arrays: The role of quantum interference on direction-dependent photon statistics
}

\author{
Iñigo Liberal, ${ }^{1,2, *}$ Iñigo Ederra, ${ }^{1,2}$ and Richard W. Ziolkowski ${ }^{3,4}$ \\ ${ }^{1}$ Electrical and Electronic Engineering Department, Universidad Pública de Navarra, Campus Arrosadía, Pamplona 31006, Spain \\ ${ }^{2}$ Institute of Smart Cities, Universidad Pública de Navarra, Campus Arrosadía, Pamplona 31006, Spain \\ ${ }^{3}$ Department of Electrical and Computer Engineering, University of Arizona, Tucson, Arizona 85721, USA \\ ${ }^{4}$ Global Big Data Technologies Centre, University of Technology Sydney, Ultimo, NSW 2007, Australia
}

(Received 10 January 2018; published 31 May 2018)

\begin{abstract}
We investigate the role of quantum interference phenomena on the characteristics of the fields radiated by an array of quantum emitters. In analogy to, but distinct from, classical outcomes, we demonstrate that the array geometry empowers control over direction-dependent photon statistics of arbitrary order. Our formulation enables the recognition of configurations providing spatial correlations with no classical counterpart. For example, we identify a system in which the angular distribution of the average number of photons is independent of the number and position of the emitters, while its higher-order photon statistics exhibit a directional behavior. These results extend our understanding of the fields generated by ensembles of quantum emitters, with potential applications to nonclassical light sources.
\end{abstract}

DOI: 10.1103/PhysRevA.97.053847

\section{INTRODUCTION}

Controlling the emission and interaction properties of an ensemble of quantum emitters plays a central role in quantum optics. The interactions between the emitters generally enrich the response of the system, leading to the emergence of collective phenomena such as superradiance [1,2], subradiance [3], collective Lamb shift [4,5], boosting strong coupling to an optical mode [6], and preserving antibunching in large samples [7]. Consequently, arrays of quantum emitters find applications to a variety of quantum engineering applications [8-11]. The geometry of the arrays in these systems is utilized to tune the nature and the strength of the interactions between the individual quantum emitters. Recent advances on the deterministic fabrication of quantum emitter arrays, including one-dimensional [12] and two-dimensional [13] arrays of cold atoms, color centers in arrays of dielectric pillars [14], and atomically thin semiconductors on top of patterned surfaces [15], are expected to push forward further experimental efforts, as well as to reveal new possibilities.

It is well known that classical antenna arrays enable shaping and reconfiguring the emitted power pattern beyond the capabilities of their single antenna elements [16-19]. In essence, by controlling the position of the radiators and the magnitude and phase of the signals driving them, one can direct the overall emission towards a preferred direction, or even dynamically scan it. Antenna arrays are nowadays a well-established technology, with applications ranging from small handset communication devices to large radar systems and radiotelescopes [20]. In contrast with arrays of quantum emitters, classical antenna arrays are primarily based on interference processes. The interaction between the individual antenna elements in the array is usually referred to as the coupling between the elements. It is often considered to be a hard factor to control or even a detrimental factor since it can induce negative outcomes such as scan blindness [19]. In

*Corresponding author: inigo.liberal@unavarra.es many cases, the coupling between the emitters can be reduced with the use of electromagnetic band gap structures [21,22] or metastructures [23] and/or compensated with reconfigurable feeding networks [17] and signal-processing techniques [24].

The conceptual differences between arrays of classical and quantum emitters has motivated us to propose the concept of quantum antenna arrays, i.e., geometrical arrangements of quantum radiators, whose emission properties are primarily designed based on quantum interference processes. As we will demonstrate, the degrees of freedom and functionalities of quantum antenna arrays far exceed those of their classical counterparts. This is due to the fact that not only the emitted intensity, but also any photon statistic can be controlled by adjusting the geometry of the array.

\section{THEORY OF QUANTUM ANTENNA ARRAYS}

As schematically depicted in Fig. 1, we start by considering a generic array of $N$ coherent quantum emitters, each modeled as a two-level $\left\{\left|e_{n}\right\rangle,\left|g_{n}\right\rangle\right\}$ system located at position $\mathbf{r}_{n}$ with transition frequency $\omega_{n}$ and with its dipole moment aligned along the $Z$ axis, $\mathbf{p}_{n}=\mathbf{u}_{z} p_{n}$. The source electric field operator (positive frequency component), $\widehat{\mathbf{E}}^{(+)}(\mathbf{r}, t)=\mathbf{u}_{\theta} \widehat{E}_{\theta}^{(+)}(\mathbf{r}, t)$, can be written as follows [25-27] (see also Appendix A):

$$
\widehat{E}_{\theta}^{(+)}(\mathbf{r}, t)=\frac{\mu_{0}}{4 \pi} \frac{\sin \theta}{r} \sum_{n=1}^{N} p_{n} \partial_{t}^{2} \widehat{\sigma}_{n}\left(t_{d}+\frac{\mathbf{u}_{r} \cdot \mathbf{r}_{n}}{c}\right),
$$

where $t_{d}=t-r / c$ is the delayed time with respect to the origin of the coordinates, and $\widehat{\sigma}_{n}(t)$ is the annihilation operator associated with the $n$th emitter. The emitters will in general exhibit complex dynamics due to their mutual interactions. However, here we are interested in the quantum interference phenomena originating from their geometrical arrangement.

In order to emphasize these aspects, we will restrict our analysis to sparse arrays where those couplings between the emitters reduce to a small perturbation. Then we can safely 


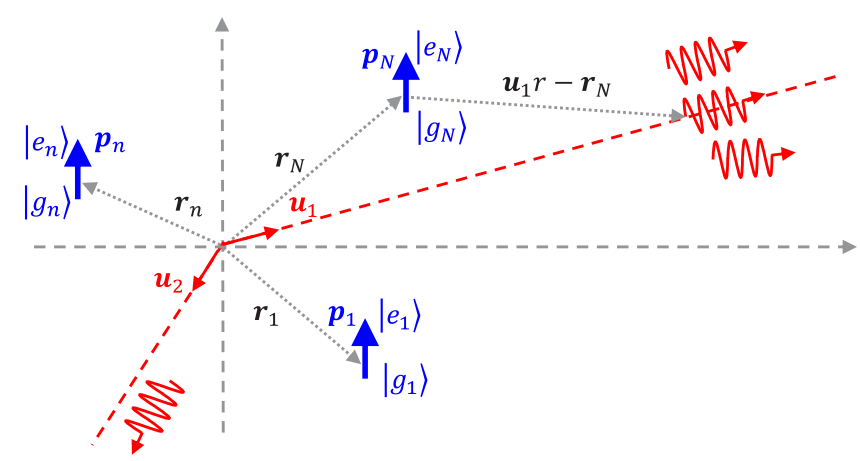

FIG. 1. Conceptual sketch of the system: an array of $N$ quantum emitters, modeled as two-level $\left\{\left|e_{n}\right\rangle,\left|g_{n}\right\rangle\right\}$ systems with dipole moments $\mathbf{p}_{n}$, located at positions $\mathbf{r}_{n}$, that emit photons into different directions, $\mathbf{u}_{q}$ and have photon statistics which depend on the geometry of the array.

assume that the individual emitter dynamics are described via an exponential decay: $\widehat{\sigma}_{n}(t) \simeq e^{-i \omega_{n} t} e^{-\gamma_{n} t} \widehat{\sigma}(t=0)$, which yields $\partial_{t}^{2} \widehat{\sigma}_{n}(t) \simeq-\omega_{n}^{2} \widehat{\sigma}_{n}(t)$, with $\gamma_{n}$ being the decay rate of the $n$th emitter (see Appendix B for a discussion on the accuracy of this approximation). This regime brings the outcomes closer to those from classical antenna arrays, where interference is the main mechanism in tailoring the far-field emission properties. Within this regime Eq. (1) reduces to

$$
\widehat{E}_{\theta}^{(+)}(\mathbf{r}, t)=\frac{\sin \theta}{r} \sum_{n=1}^{N} B_{n} \widehat{\sigma}_{n} e^{-\left(i \omega_{n}+\gamma_{n}\right) t_{d}} e^{-i \frac{\omega_{n}}{c} \mathbf{u}_{r} \cdot \mathbf{r}_{n}}
$$

with $B_{n}=-\mu_{0} \omega_{n}^{2} p_{n} /(4 \pi)$. We omit here and below the $(t=0)$ labels in the atomic operators to simplify notations.

Next, we investigate the impact of the array geometry within different photon statistics. Following Glauber's seminal work [28,29], we compute the probability densities $P_{L}\left(\mathbf{u}_{1}, t_{1} ; \ldots ; \mathbf{u}_{L}, t_{L}\right)$ per unit (time) ${ }^{L}$ per (solid angle) ${ }^{L}$ of measuring $L$ photons at times $t_{1}, \ldots, t_{L}$ and in directions $\mathbf{u}_{1}, \ldots, \mathbf{u}_{L}$, as being proportional to the field correlation functions

$$
P_{L} \propto r^{2 L}\left\langle\widehat{E}_{\theta, 1}^{(-)} \cdots \widehat{E}_{\theta, L}^{(-)} \widehat{E}_{\theta, L}^{(+)} \cdots \widehat{E}_{\theta, 1}^{(+)}\right\rangle
$$

with $\widehat{E}_{\theta, 1}^{(-)}=\widehat{E}_{\theta}^{(-)}\left(\mathbf{r}_{1}, t_{1}\right)$ and so on. Note that any other photon statistic can be constructed from these probability densities [30,31]. In general, the probability density $P_{L}\left(\mathbf{u}_{1}, t_{1} ; \ldots ; \mathbf{u}_{L}, t_{L}\right)$ described by Eq. (3) leads to interesting spatiotemporal correlations. However, here we are considering decoupled emitters, for which their time evolution is uncorrelated. As a result, the time evolution of Eq. (3) is characterized by $\exp \left[i\left(\omega_{n_{l}}-\omega_{m_{l}}\right) t_{l}\right]$ factors that lead to an oscillatory behavior of the angular properties of the photon statistics, as well as to $\exp \left[-\left(\gamma_{n_{l}}+\gamma_{m_{l}}\right) t_{l}\right]$ factors that lead to an overall exponential decay. In order to emphasize the directional behavior of the emissions, irrespective of the details associated with their times of arrival, we define here the time integral of these probability densities:

$$
g_{L}\left(\mathbf{u}_{1}, \ldots, \mathbf{u}_{L}\right)=\prod_{l=1}^{L} \int_{0}^{\infty} d t_{l} P_{L}\left(\mathbf{u}_{1}, t_{1} ; \ldots ; \mathbf{u}_{L}, t_{L}\right)
$$

The functions (4) include time integrals over rapidly oscillatory functions that are related to the transition frequency of the dipoles, $\omega_{n}$. Unless the transition frequencies are very close to each other, these integrals will average to zero over time, and interference phenomena might only be observable for short time intervals. In general, this property indicates that no quantum interference of any significance will take place unless the emitters have similar transition frequencies. Hereafter, we assume all emitters to be identical $\left(\omega_{n}=\omega_{0}, p_{n}=p \forall n\right)$. Then defining the $L$-order generalized quantum array factor:

$$
\begin{gathered}
f_{L}\left(\mathbf{u}_{1}, \ldots, \mathbf{u}_{L}\right)=\sum_{n_{1}, \ldots, n_{L}=1}^{N} \sum_{m_{1}, \ldots, m_{L}=1}^{N} \\
\left\langle\widehat{\sigma}_{n_{1}}^{\dagger} \ldots \widehat{\sigma}_{n_{L}}^{\dagger} \widehat{\sigma}_{m_{L}} \ldots \widehat{\sigma}_{m_{1}}\right\rangle \prod_{p=1}^{L} e^{i k_{0} \mathbf{u}_{p} \cdot \mathbf{r}_{n_{p}}} \prod_{q=1}^{L} e^{-i k_{0} \mathbf{u}_{q} \cdot \mathbf{r}_{m_{q}}} .
\end{gathered}
$$

Equation (4) reduces to

$$
g_{L}\left(\mathbf{u}_{1}, \ldots, \mathbf{u}_{L}\right) \propto A^{L}\left(\prod_{l=1}^{L} \sin ^{2} \theta_{l}\right) f_{L}\left(\mathbf{u}_{1}, \ldots, \mathbf{u}_{L}\right)
$$

with $A=\left(\frac{\mu_{0}}{4 \pi} \omega_{0}^{2} p\right)^{2} /(2 \gamma)$. The $g_{L}$ integrated correlations represent the average number of $L$-photon coincidence measurements for any time delay. In the next sections, we will focus on the first- and second-order correlations. For the sake of completeness, the generalization of this theory to arrays of nonidentical emitters with variable polarization is reported in Appendix C.

\section{FIRST-ORDER CORRELATIONS}

The first-order correlation, $g_{1}\left(\mathbf{u}_{1}\right) \propto A \sin \theta_{1} f_{1}\left(\mathbf{u}_{1}\right)$ represents the average number of photons measured by a detector placed along the direction $\mathbf{u}_{1}$. The associated first-order quantum array factor can be written as

$$
f_{1}\left(\mathbf{u}_{1}\right)=\sum_{n, m=1}^{N}\left\langle\widehat{\sigma}_{n}^{\dagger} \widehat{\sigma}_{m}\right\rangle e^{i k \mathbf{u}_{1} \cdot \mathbf{r}_{n}} e^{-i k \mathbf{u}_{1} \cdot \mathbf{r}_{m}}
$$

It is clear from (7) that the first-order generalized quantum array factor is very similar to the magnitude squared of the classical array factor [16-19], with the initial-time correlation $\left\langle\widehat{\sigma}_{n}^{\dagger} \widehat{\sigma}_{m}\right\rangle$ playing a role similar to that of the product of the signals feeding the $n$th and $m$ th radiating elements. However, there is also an important difference that induces nonclassical effects even at the average number of photons level. Specifically, the initial-time correlation is not always necessarily factored as the product of two c-numbers, e.g., as $\left\langle\widehat{\sigma}_{n}^{\dagger} \widehat{\sigma}_{m}\right\rangle=a_{n}^{*} a_{m}$. As a consequence, access is granted to configurations forbidden in the classical case.

One then wonders if these extra degrees of freedom enable an increase of the directivity, i.e., in the average number of photons in a specified direction, beyond the classical limits. It can be demonstrated that this is not the case. Because the initialtime correlation has some restrictions, e.g., a matrix formed by elements $\left\langle\sigma_{n}^{\dagger} \sigma_{m}\right\rangle$ must be Hermitian and positive semidefinite, it is possible to prove formally that the directivity cannot be increased beyond the classical limit (see Appendix D). 
For any given array geometry, very different angular responses will be obtained as a function of the initial state of the system, and different experimental techniques could be applied for the preparation of any initial state of the system. Initialization laser pulses can be employed for initial states corresponding to product states (see, e.g., Ref. [32]), while more complex entangled states with a specific phase profile could be implemented with laser-assisted interactions [33,34]. We take here, for example, a single-excitation initial state $\left|\psi_{1}\right\rangle=\sum_{r=1}^{N} a_{r} \widehat{\sigma}_{r}^{\dagger}|0\rangle$, for which we recover a factorization analogous to the classical case $\left\langle\widehat{\sigma}_{n}^{\dagger} \widehat{\sigma}_{m}\right\rangle=a_{n}^{*} a_{m}$. Subsequently, the first-order generalized array factor reduces to

$$
f_{1, \psi_{1}}\left(\mathbf{u}_{1}\right)=\left|\sum_{m=1}^{N} a_{m} e^{-i k_{0} \mathbf{u}_{1} \cdot \mathbf{r}_{m}}\right|^{2} .
$$

Therefore, it can be concluded that with the decay of a single excitation, the radiated field can be beam formed exactly as in the classical case, the probability amplitudes playing a role analogous to the currents driving the classical antenna elements. This is an exciting outcome because it means that all the machinery developed for the synthesis of classical antenna arrays can be directly applied to single-excitation states.

Nevertheless, configurations without a classical counterpart are of even greater interest. For example, in the case in which all $N$ emitters are initially excited, i.e., with $\left|\psi_{S N}\right\rangle=\prod_{r=1}^{N} \widehat{\sigma}_{r}^{\dagger}|0\rangle$, we find $\left\langle\widehat{\sigma}_{n}^{\dagger} \widehat{\sigma}_{m}\right\rangle=\delta_{n m}$, and hence

$$
f_{1, \psi_{S N}}=N .
$$

This result implies that the emitters do not interfere and, hence, do not modify the directional properties of the average number of photons. In other words, the emission pattern in terms of the average number of photons is identical to the individual emitter pattern, $a \sin ^{2} \theta$ function, independent of the number and geometrical arrangement of the emitters. This is a quantum effect with no counterpart in classical antenna array theory.
Many other states can be studied. For example, the initialtime correlation function for a symmetric two-excitation state, i.e., for $\left|\psi_{S 2}\right\rangle=\sum_{r=1}^{N-1} \sum_{s=r+1}^{N} \widehat{\sigma}_{r}^{\dagger} \widehat{\sigma}_{s}^{\dagger}|0\rangle / \sqrt{C_{2}}$, with normalization constant $C_{2}=N(N-1) / 2$, is given by $\left\langle\widehat{\sigma}_{n}^{\dagger} \widehat{\sigma}_{m}\right\rangle=$ $C_{2}^{-1}\left((N-2)+\delta_{n m}\right)$. The first-order generalized array factor is then given by

$$
f_{1, \psi_{s 2}}\left(\mathbf{u}_{1}\right)=\frac{2}{(N-1)}+\frac{2(N-2)}{N(N-1)}\left|\sum_{m=1}^{N} e^{-i k_{0} \mathbf{u}_{1} \cdot \mathbf{r}_{m}}\right|^{2} .
$$

It can then be concluded that this initial state also exhibits directional properties in the average number of photons which corresponds to a weighted average of the single-excitation and $N$-excitation state responses.

As an illustrative example, Fig. 2 gathers the $g_{1}\left(\mathbf{u}_{1}\right)$ angular patterns for a linear, vertical array of $N=3$ emitters, each emitter separated from another by one wavelength, i.e., for the emitter locations $\mathbf{r}_{n}=\mathbf{u}_{z} n \lambda_{0}$. The geometry is rotationally symmetric around the $Z$ axis and, hence, the patterns can be plotted simply in the $X Z$ plane. This is a canonical antenna array configuration that enhances the directivity by channeling the emitted radiation into the azimuthal plane $(\theta=\pi / 2)$ [16]. Figure 2 shows that the emission directivity is indeed enhanced for the single- and two-excitation states, i.e., the emitted photons will be constrained to an narrower set of directions than in the single emitter case (shown as a dashed black line). In contrast, the angular pattern for the case in which all three emitters are initially excited is identical to that of the single emitter. This outcome confirms the peculiar effect that the directionality on the average number of photons in the case in which all the identical emitters are excited is not affected by their array configuration. However, in general, the geometry of the array will have an impact on the photon statistics of any order. Moreover, the angular patterns of different-order photon correlations can have qualitatively different properties. In order to illustrate this effect, in the next section we address the second order correlation for this system.

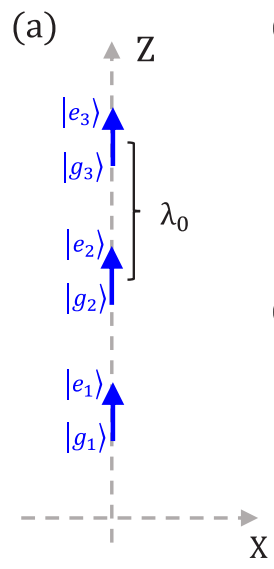

(b)
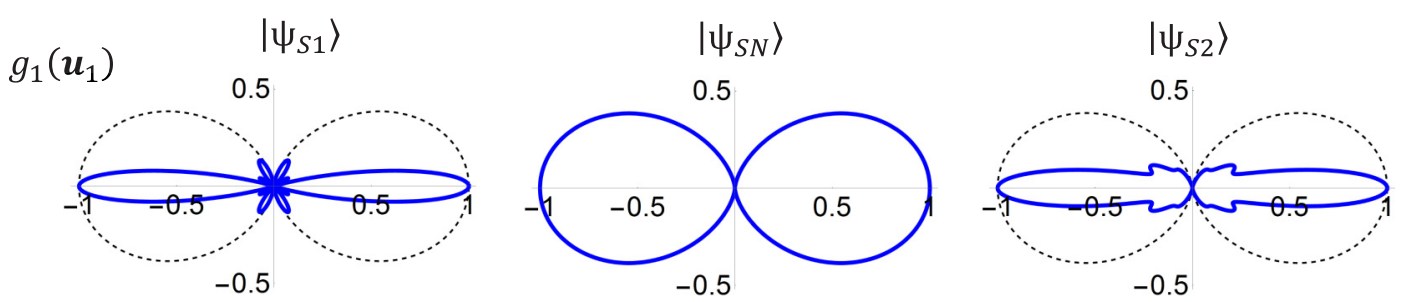

(c)
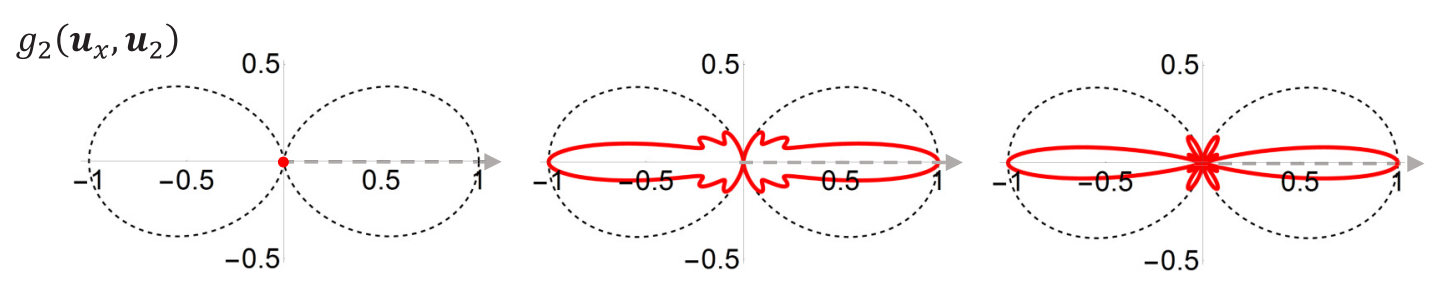

FIG. 2. Linear array of $N=3$ quantum emitters located along the $Z$ axis with constant separation $d=\lambda_{0}$. (a) Sketch of the geometry. (b) First-order correlation patterns, $g_{1}\left(\mathbf{u}_{1}\right)$ as a function of $\theta_{1}$ for three different initial states: symmetrical single-excitation state $\left|\psi_{S 1}\right\rangle$, symmetrical $N$-excitation state $\left|\psi_{S N}\right\rangle$, and symmetrical two-excitation state $\left|\psi_{S 2}\right\rangle$. (c) Same as in (b) but for the second-order correlation function $g_{1}\left(\mathbf{u}_{1}, \mathbf{u}_{2}\right)$ as a function of $\mathbf{u}_{2}$ for $\mathbf{u}_{1}=\mathbf{u}_{x}$ (indicated as a gray dashed arrow). 


\section{SECOND-ORDER CORRELATIONS}

The second-order time integrated correlation is given by: $g_{2}\left(\mathbf{u}_{1}, \mathbf{u}_{2}\right) \propto \sin \theta_{1} \sin \theta_{2} f_{2}\left(\mathbf{u}_{1}, \mathbf{u}_{2}\right)$. It represents the average number of two-photon coincidences for any time delay. This is a good figure of merit of how directionally bunched the emitted photons are. Specifically, since Glauber's probability densities are nonexclusive quantities [30], $g_{2}\left(\mathbf{u}_{1}, \mathbf{u}_{2}\right)=0$ implies that no photon coincidences of any order will be recorded along the directions $\mathbf{u}_{1}$ and $\mathbf{u}_{2}$ for the same decay process. Consequently, a highly directive pattern of $g_{2}\left(\mathbf{u}_{1}, \mathbf{u}_{2}\right)$ indicates that all emitted photons are bunched into a narrow set of directions.

Similar to the first-order array factor, the second-order array factor $f_{2}\left(\mathbf{u}_{1}, \mathbf{u}_{2}\right)$ is determined by the initial-time correlation function, $\left\langle\sigma_{n_{1}}^{\dagger} \sigma_{n_{2}}^{\dagger} \sigma_{m_{2}} \sigma_{m_{1}}\right\rangle$, and the phase factors associated with the position of the emitters. However, the angular patterns of the second-order correlations are essentially different from those of the first-order correlations. For example, Fig. 2(c) depicts $g_{2}\left(\mathbf{u}_{1}, \mathbf{u}_{2}\right)$ for $\mathbf{u}_{1}=\mathbf{u}_{x}$ as a function of $\mathbf{u}_{2}$, for the configuration studied in Fig. 2(b). In particular, for the singleexcitation state we trivially obtain $\left\langle\sigma_{n_{1}}^{\dagger} \sigma_{n_{2}}^{\dagger} \sigma_{m_{2}} \sigma_{m_{1}}\right\rangle=0$, which means $g_{2}\left(\mathbf{u}_{1}, \mathbf{u}_{2}\right)=0 \forall \mathbf{u}_{1}, \mathbf{u}_{2}$. This nonclassical effect, analogous to antibunching, simply relates to the impossibility of measuring two excitations from a single-excitation state (i.e., recall that higher-order multiphoton processes are disregarded in our model).

Interestingly, for the $N$-excitation state we obtain $\left\langle\sigma_{n_{1}}^{\dagger} \sigma_{n_{2}}^{\dagger} \sigma_{m_{2}} \sigma_{m_{1}}\right\rangle=\left(1-\delta_{m_{1} m_{2}}\right)\left(\delta_{n_{1} m_{1}} \delta_{n_{2} m_{2}}+\delta_{n_{1} m_{2}} \delta_{n_{2} m_{1}}\right)$. Then the second-order generalized array factor can be written as

$$
f_{2, \psi_{S N}}\left(\mathbf{u}_{1}, \mathbf{u}_{2}\right)=N(N-2)+\left|\sum_{m=1}^{N} e^{-i k_{0}\left(\mathbf{u}_{1}-\mathbf{u}_{2}\right) \cdot \mathbf{r}_{m}}\right|^{2} .
$$

This nontrivial correlation leads to a directional behavior in $g_{2}\left(\mathbf{u}_{1}, \mathbf{u}_{2}\right)$, despite the absence of any directionality in $g_{1}\left(\mathbf{u}_{1}\right)$. We find in this manner that the photons are probabilistically emitted with no directional preference other than the pattern of the single emitter, i.e., with no directionality for $g_{1}\left(\mathbf{u}_{1}\right)$. On the other hand, for a given decay process they will be all measured bunched around a set of directions, i.e., with the directionality of $g_{2}\left(\mathbf{u}_{1}, \mathbf{u}_{2}\right)$.

This effect is somewhat analogous to two-photon interference in beam splitters. The two photons are equally probable to exit this system into any of the two output ports. However, both are certain to exit into the same output port [25]. Here a number $N$ of photons are equally probable (up to the individual emitter pattern) to exit the system on a continuum of output channels (i.e., the directions), but they are certain to be measured within a narrow interval of directions. This effect can be more clearly appreciated in Fig. 3. The second-order correlation $g_{2}\left(\mathbf{u}_{1}, \mathbf{u}_{2}\right)$ is presented for three different $\mathbf{u}_{1}$ given by $\left(\phi_{1}=\right.$ $\left.0, \theta_{1}=0.3 \pi\right),\left(\phi_{1}=0, \theta_{1}=0.4 \pi\right)$, and $\left(\phi_{1}=0, \theta_{1}=0.5 \pi\right)$. The angular patterns consist of lobes centered around each $\mathbf{u}_{1}$, plus additional grating lobes.

These photon statistic outcomes can then be tailored by changing the geometry of the array. To this end, different design techniques could be developed, which are likely to exhibit similarities and differences with respect to synthesis techniques of classical antenna arrays. In order to illustrate this point, Fig. 4 depicts $g_{2}\left(\mathbf{u}_{1}, \mathbf{u}_{2}\right)$ as a function of the number

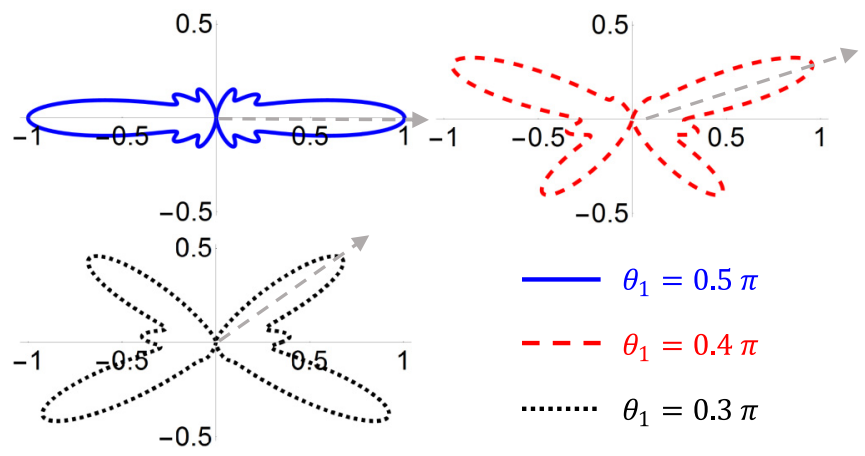

FIG. 3. Normalized second-order angular correlation function $g_{2}\left(\mathbf{u}_{1}, \mathbf{u}_{2}\right)$ evaluated for a vertical linear array of $N=$ 3 emitters, with all emitters initially excited, when $\mathbf{u}_{1}\left(\phi_{1}=0\right.$, $\left.\theta_{1}=0.5 \pi\right), \mathbf{u}_{1}\left(\phi_{1}=0, \theta_{1}=0.4 \pi\right)$, and $\mathbf{u}_{1}\left(\phi_{1}=0, \theta_{1}=0.3 \pi\right)$. Gray dashed arrow indicates the direction of $\mathbf{u}_{1}$ in each subpanel.

of emitters and the distance of separation between them. In analogy with classical antenna arrays, we find that increasing the separation between the emitters results in the appearance of grating lobes, i.e., the emitted photons will be bunched around not one, but a set of multiple lobes. However, in contrast with classical antenna arrays, it is found that the directivity does not increase monotonically along with the number of emitters. On the contrary, the $g_{2}\left(\mathbf{u}_{1}, \mathbf{u}_{2}\right)$ results in the superposition of the single emitter pattern with that of a highly directive lobe. These results suggest that nonclassical techniques will have to be developed for the synthesis of quantum antenna arrays and each of their different photon correlations.
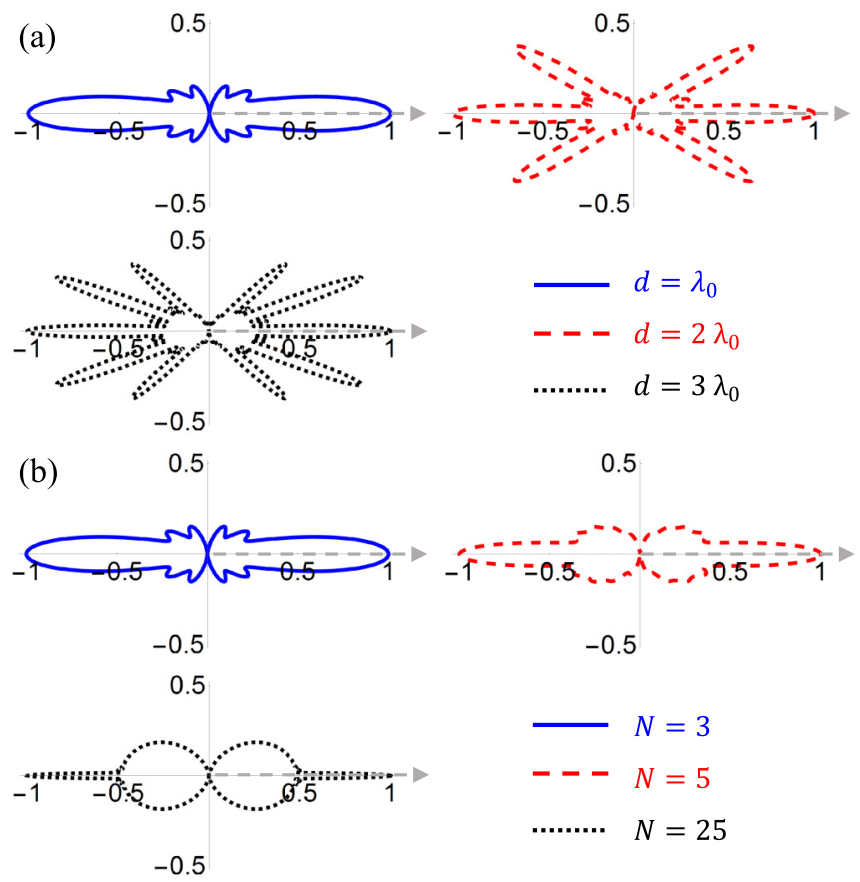

FIG. 4. Normalized second-order angular correlation function $g_{2}\left(\mathbf{u}_{1}, \mathbf{u}_{2}\right)$ evaluated with $\mathbf{u}_{1}\left(\phi_{1}=0, \theta_{1}=0.5 \pi\right)$ for a linear vertical array of (a) $N=3$ elements with separation distances $d=1 \lambda_{0}, d=$ $2 \lambda_{0}$, and $d=3 \lambda_{0}$, and (b) a separation distance $d=1 \lambda_{0}$ and arrays with $N=3, N=5$, and $N=25$ emitters. Gray dashed arrow indicates the direction of $\mathbf{u}_{1}$ in each subpanel. 
To finalize the discussion, we come back to the twoexcitation state, with initial-time second-order correlation function: $\left\langle\widehat{\sigma}_{n_{1}}^{\dagger} \widehat{\sigma}_{n_{2}}^{\dagger} \widehat{\sigma}_{m_{2}} \widehat{\sigma}_{m_{1}}\right\rangle=\left(1-\delta_{n_{1} n_{2}}\right)\left(1-\delta_{m_{1} m_{2}}\right)$. Subsequently, the associated second-order array factor can be compactly written as

$f_{2, \psi_{S 2}}\left(\mathbf{u}_{1}, \mathbf{u}_{2}\right)=\frac{1}{C_{2}}\left|\sum_{n, m=1}^{N}\left(1-\delta_{n m}\right) e^{-i k_{0} \mathbf{u}_{2} \cdot \mathbf{r}_{n}} e^{-i k_{0} \mathbf{u}_{1} \cdot \mathbf{r}_{m}}\right|^{2}$.

Figure 2(c) depicts the $g_{2}\left(\mathbf{u}_{1}, \mathbf{u}_{2}\right)$ pattern for $\mathbf{u}_{1}=\mathbf{u}_{x}$ for this state. We find that the second-order correlations for the two-excitation state exhibit directional properties similar to the first-order correlation. This implies that the photons are not bunched beyond the directional properties imposed by the average number of photons.

\section{CONCLUSIONS}

Our results have theoretically demonstrated that it is possible to engineer the angular properties of different photon statistics by adjusting the geometry of an array of quantum emitters. Our methodology highlights the role that quantum interference processes play rather than the interactions between the emitters. As a consequence, we have linked the concepts of arrays of quantum emitters and classical antenna arrays, i.e., we have presented results for quantum antenna arrays. The proposed formulation, based on a generalized array factor, facilitates the analysis of these systems. By using it we were able to demonstrate that the directivity in the average number of photons cannot beat the classical limit, as well as to identify configurations with no classical counterpart. For example, we demonstrated that there is an initial state for which the emission pattern in terms of the average number of photons is independent of the number and position of the emitters. We further demonstrated that higher-order correlations also exhibit directivity even in that case. These outcomes suggest that these quantum antenna arrays can be used as sources of directionally entangled photon bunches.

Further evolution of these concepts can be envisioned on the basis of previous experiences with classical antenna arrays. Many configurations could be studied, including multiple array topologies, their interaction with nanophotonic structures, as well as the analysis of additional correlation functions. We believe that these results expand our current understanding of the emission properties of ensembles of quantum emitters, and they might also find applications as nonclassical light sources.

\section{ACKNOWLEDGMENT}

I.L. acknowledges support from a Juan de la Cierva Incorporación Fellowship and Dotación Adicional Caixa.

\section{APPENDIX A: SOURCE FIELD IN THE FAR ZONE}

Here we provide the derivation of Eq. (1), i.e., the expression of the positive frequency source electric field operator. For the sake of completeness, we assume that the array of quantum emitters are coupled to a common macroscopic photonic environment, characterized by the relative permittivity, $\varepsilon\left(\mathbf{r}, \omega_{f}\right)=$ $\varepsilon_{R}\left(\mathbf{r}, \omega_{f}\right)+i \varepsilon_{I}\left(\mathbf{r}, \omega_{f}\right)$, and modeled as a bath of polaritonic modes following the macroscopic quantum electrodynamics (QED) formalism (see, e.g., Ref. [35]). This approach allows us to keep the generality for an array located in an arbitrary photonic environment. Later on we take free space as a limiting case as is usually done in this formalism (see, e.g., Ref. [36] and the references therein).

Within this model, the Hamiltonian of the system in the Heisenberg picture is given by $\widehat{\mathcal{H}}=\widehat{\mathcal{H}}_{0}+\widehat{\mathcal{H}}_{I}$ with $\widehat{\mathcal{H}}_{0}=\sum_{n=1}^{N} \frac{\hbar \omega_{n}}{2} \widehat{\sigma}_{n}^{z}(t)+\int d^{3} \mathbf{r} \int_{0}^{\infty} d \omega_{f} \hbar \omega_{f} \widehat{\mathbf{f}}^{\dagger}\left(\mathbf{r}, \omega_{f} ; t\right)$. $\widehat{\mathbf{f}}\left(\mathbf{r}, \omega_{f} ; t\right)$ and $\widehat{\mathcal{H}}_{I}=-\sum_{n=1}^{N} \widehat{\mathbf{p}}_{n}(t) \cdot \widehat{\mathbf{E}}\left(\mathbf{r}_{n} ; t\right)$. Here $\widehat{\mathbf{f}}\left(\mathbf{r}, \omega_{f} ; t\right)$ are polaritonic annihilation operators representing excitations of the field-matter system, obeying the equal-time commutation relation $\left[\widehat{\mathbf{f}}\left(\mathbf{r}, \omega_{f} ; t\right), \widehat{\mathbf{f}}\left(\mathbf{r}^{\prime}, \omega_{f}^{\prime} ; t\right)\right]=\widehat{\mathbf{I}} \delta\left(\mathbf{r}-\mathbf{r}^{\prime}\right) \delta\left(\omega_{f}-\omega_{f}^{\prime}\right)$, and $\widehat{\sigma}_{n}^{z}, \widehat{\sigma}_{n}$ are the usual Pauli operators for the two-level systems. Similarly, $\widehat{\mathbf{p}}_{n}(t)=\mathbf{p}_{n} \widehat{\sigma}_{n}(t)+\mathbf{p}_{n}^{*} \widehat{\sigma}_{n}^{\dagger}(t)$ is the electric dipole operator, and the electric field operator is given by

$$
\widehat{\mathbf{E}}(\mathbf{r} ; t)=\int_{0}^{\infty} d \omega_{f} \int d^{3} \mathbf{r}^{\prime} \mathbf{G}_{E}\left(\mathbf{r}, \mathbf{r}^{\prime}, \omega_{f}\right) \cdot \widehat{\mathbf{f}}\left(\mathbf{r}^{\prime}, \omega_{f} ; t\right)+\text { H.c. }
$$

where we have introduced the response function

$$
\mathbf{G}_{E}\left(\mathbf{r}, \mathbf{r}^{\prime}, \omega_{f}\right)=i \sqrt{\frac{\hbar}{\pi \varepsilon_{0}}} \frac{\omega_{f}^{2}}{c^{2}} \sqrt{\varepsilon_{I}\left(\mathbf{r}^{\prime}, \omega_{f}\right)} \mathbf{G}\left(\mathbf{r}, \mathbf{r}^{\prime}, \omega_{f}\right),
$$

which is proportional to the classical dyadic Green's function $\mathbf{G}\left(\mathbf{r}, \mathbf{r}^{\prime}, \omega_{f}\right)$.

Next we compute the Heisenberg equation of motion, $i \hbar \partial_{t} \widehat{O}(t)=[\widehat{O}, \widehat{\mathcal{H}}]$, for the polaritonic operator $\widehat{\mathbf{f}}\left(\mathbf{r}^{\prime}, \omega_{f} ; t\right)$, and integrate it, leading to its decomposition into the freeevolving and source fields associated with each quantum emitter

$$
\widehat{\mathbf{f}}\left(\mathbf{r}^{\prime}, \omega_{f} ; t\right)=\widehat{\mathbf{f}}_{0}\left(\mathbf{r}^{\prime}, \omega_{f} ; t\right)+\sum_{n=1}^{N} \widehat{\mathbf{f}}_{S n}\left(\mathbf{r}^{\prime}, \omega_{f} ; t\right)
$$

with the definitions

$$
\begin{gathered}
\widehat{\mathbf{f}}_{0}\left(\mathbf{r}^{\prime}, \omega_{f} ; t\right)=\widehat{\mathbf{f}}\left(\mathbf{r}^{\prime}, \omega_{f} ; t=0\right) e^{-i \omega_{f} t}, \\
\widehat{\mathbf{f}}_{S n}\left(\mathbf{r}^{\prime}, \omega_{f} ; t\right)=-\frac{1}{i \hbar} \int_{0}^{t} d \tau e^{-i \omega_{f}(t-\tau)} \widehat{\mathbf{p}}_{n}(\tau) \cdot \mathbf{G}_{E}^{*}\left(\mathbf{r}_{n}, \mathbf{r}^{\prime}, \omega_{f}\right) .
\end{gathered}
$$

Similarly, we can decompose the electric field operator into the free-evolving and source parts

$$
\widehat{\mathbf{E}}(\mathbf{r} ; t)=\widehat{\mathbf{E}}_{0}(\mathbf{r} ; t)+\sum_{n=1}^{N} \widehat{\mathbf{E}}_{S n}(\mathbf{r} ; t) .
$$

By using the completeness relation of the dyadic Green's function, as well as the fact that $\operatorname{Im} \mathbf{G}\left(\mathbf{r}, \mathbf{r}_{n}, \omega_{f}\right)$ is an odd function in $\omega_{f}$, the contribution of each emitter to the source field can be written as a memory kernel acting of the electric dipole operator

$$
\widehat{\mathbf{E}}_{S n}(\mathbf{r} ; t)=\int_{0}^{t} d \tau \mathbf{K}\left(\mathbf{r}, \mathbf{r}_{n}, t-\tau\right) \cdot \widehat{\mathbf{p}}_{n}(\tau)
$$

with

$$
\mathbf{K}\left(\mathbf{r}, \mathbf{r}_{n}, t\right)=i \int_{-\infty}^{\infty} d \omega_{f} e^{-i \omega_{f} t} \frac{\omega_{f}^{2}}{\pi \varepsilon_{0} c^{2}} \operatorname{Im} \mathbf{G}\left(\mathbf{r}, \mathbf{r}_{n}, \omega_{f}\right) .
$$


We can then apply the Laplace transform to the operators, $\widehat{O}(\omega)=\int_{0}^{\infty} d \omega \widehat{O}(t) e^{i \omega t}$, so that Eq. (A7) can be more conveniently rewritten as

$$
\widehat{\mathbf{E}}_{S n}(\mathbf{r} ; \omega)=\omega^{2} \mu_{0} \mathbf{G}\left(\mathbf{r}, \mathbf{r}_{n}, \omega\right) \cdot \widehat{\mathbf{p}}_{n}(\omega) .
$$

Next, we recall the decomposition of the electric dipole operator as $\widehat{\mathbf{p}}_{n}(\tau)=\mathbf{p}_{n} \widehat{\sigma}_{n}(t)+\mathbf{p}_{n}^{*} \widehat{\sigma}_{n}^{\dagger}(t)$, which justifies the decomposition of the source electric field operator into positive and negative frequency components. The latter are related to the annihilation or creation of an electronic excitation at the $n$th quantum emitter $\widehat{\mathbf{E}}_{S n}(\mathbf{r} ; t)=\widehat{\mathbf{E}}_{S n}^{(+)}(\mathbf{r} ; t)+\widehat{\mathbf{E}}_{S n}^{(-)}(\mathbf{r} ; t)$, with $\widehat{\mathbf{E}}_{S n}^{(-)}(\mathbf{r} ; t)=\left[\widehat{\mathbf{E}}_{S n}^{(+)}(\mathbf{r} ; t)\right]^{\dagger}$, and

$$
\widehat{\mathbf{E}}_{S n}^{(+)}(\mathbf{r} ; t)=\int_{0}^{t} d \tau \mathbf{K}\left(\mathbf{r}, \mathbf{r}_{n}, t-\tau\right) \cdot \mathbf{p}_{n} \widehat{\sigma}_{n}(t) .
$$

Similarly, we can write the Laplace transform version of the positive frequency component as

$$
\widehat{\mathbf{E}}_{S n}^{(+)}(\mathbf{r} ; \omega)=\omega^{2} \mu_{0} \mathbf{G}\left(\mathbf{r}, \mathbf{r}_{n}, \omega\right) \cdot \mathbf{p}_{n} \widehat{\sigma}_{n}(\omega) .
$$

For simple comparisons with the classical antenna array outcomes, we assume that the emitters are immersed in free space and the observation point for the electric field is in the far zone of this system. In such a case, the classical dyadic Green's function reduces to

$$
\mathbf{G}\left(\mathbf{r}, \mathbf{r}_{n}, \omega\right)=\frac{e^{i k R_{n}}}{4 \pi R_{n}}\left(\mathbf{I}-\mathbf{u}_{R_{n}} \mathbf{u}_{R_{n}}\right)
$$

with $k=\omega / c, \mathbf{R}_{n}=\mathbf{r}-\mathbf{r}_{n}, R_{n}=\left|\mathbf{R}_{n}\right|$, and $\mathbf{u}_{R_{n}}=\mathbf{R}_{n} / R_{n}$. The first-order approximation to $R_{n}$ is given by $R_{n} \simeq r-\mathbf{u}_{r}$. $\mathbf{r}_{n}$. We keep the first-order approximation in the exponential and the zero-order approximation in the other terms. The dyadic Green's function then becomes

$$
\mathbf{G}\left(\mathbf{r}, \mathbf{r}_{n}, \omega\right)=\frac{1}{4 \pi r} \mathbf{I}_{T} e^{i \omega \frac{r-\mathbf{u}_{r} \cdot \mathbf{r}_{n}}{c}}
$$

with $\mathbf{I}_{T}=\mathbf{I}-\mathbf{u}_{r} \mathbf{u}_{r}$. In this manner, we can rewrite the electric field operator as

$$
\widehat{\mathbf{E}}_{S}^{(+)}(\mathbf{r} ; \omega)=\frac{\mu_{0}}{4 \pi r} \sum_{n=1}^{N} \mathbf{I}_{T} \cdot \mathbf{p}_{n} \omega^{2} \widehat{\sigma}_{n}(\omega) e^{i \omega \frac{r-u_{r} \cdot \mathbf{r}_{n}}{c}} .
$$

Finally, we apply the inverse Laplace transform and get the general form for the source electric field in the far zone

$$
\widehat{\mathbf{E}}_{S}^{(+)}(\mathbf{r} ; t)=-\frac{\mu_{0}}{4 \pi r} \sum_{n=1}^{N} \mathbf{I}_{T} \cdot \mathbf{p}_{n} \partial_{t}^{2} \widehat{\sigma}_{n}\left(t_{d}+\frac{\mathbf{u}_{r} \cdot \mathbf{r}_{n}}{c}\right)
$$

with $t_{d}=t-r / c$. In the case in which all emitters are oriented along the $Z$ axis, $\mathbf{p}_{n}=p_{n} \mathbf{u}_{z}$, we recover Eq. (1).

\section{APPENDIX B: COUPLING BETWEEN THE EMITTERS}

The presented quantum array theory is based on the approximation that the interaction between the emitters only produces a small perturbation on the dynamics of the individual emitters. In analogy with classical antenna arrays, this regime puts emphasis on quantum interference processes instead of the interaction between the emitters. It also simplifies the analysis, providing access to field correlations that would be cumbersome to compute for large arrays of strongly interacting

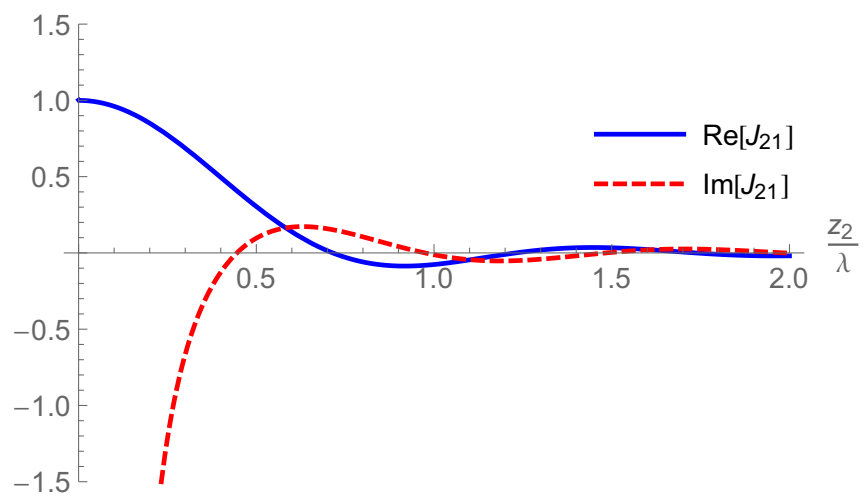

FIG. 5. Real (blue solid line) and imaginary (red dashed line) parts of the coupling parameter, $J_{21}$, normalized to $J_{11}$, as a function of the emitter separation distance, $z_{2}$.

quantum emitters. Naturally, the accuracy of the model will eventually break down as the separation between the emitters decreases. In order to discuss this point, we can consider, as a first approximation, the description of the system within the Born-Markov approximation. Consequently, the dynamics of the array can be described by a quantum master equation (QME) in Lindblad form (see, e.g., Ref. [37])

$$
\frac{d}{d t} \rho=\sum_{n, m} J_{n m}\left(\widehat{\sigma}_{n} \rho \widehat{\sigma}_{m}^{\dagger}-\rho \widehat{\sigma}_{m}^{\dagger} \widehat{\sigma}_{n}\right)+\text { H.c. }
$$

where H.c. denotes the Hermetian conjugate and we have assumed a frame rotating at the frequency of the emitters, $\omega_{0}$. The coupling parameters can then be written as [38]

$$
\begin{gathered}
J_{n m}=-i \frac{\omega_{0}^{2}}{\hbar^{2} \varepsilon_{0} c^{2}} \mathbf{p}_{n} \cdot \mathbf{G}\left(\mathbf{r}_{n}, \mathbf{r}_{m}, \omega_{0}\right) \cdot \mathbf{p}_{m}, \quad n \neq m, \\
J_{n n}=\frac{\gamma}{2}=\frac{\omega_{0}^{2}}{\hbar^{2} \varepsilon_{0} c^{2}} \mathbf{p}_{n} \cdot \operatorname{Im} \mathbf{G}\left(\mathbf{r}_{n}, \mathbf{r}_{n}, \omega_{0}\right) \cdot \mathbf{p}_{n} .
\end{gathered}
$$

Operating in the weak-coupling regime implicitly assumes that $\left|J_{n m}\right| \ll \omega_{0}$. In addition, it is clear from the formulation above that the response of the system converges to our model if the coupling parameters between the emitters are much smaller than the decay rate, $\left|J_{n m}\right| \ll \gamma$. In order to illustrate when the second condition is fulfilled, let us consider, for example, the vertical linear array studied in the examples of the main text, in which the quantum emitters are located along the $Z$ axis. Figure 5 represents the real (blue) and imaginary (red) parts of the coupling parameters for two emitters, one located at $z_{1}=0$ and another one located at $z_{2}$, normalized to $J_{11}$. On one hand, the real part of the coupling parameter converges to $J_{11}$ as the separation tends to zero. On the other hand, the imaginary part diverges in the same limit, as the reactive coupling is monotonically increased. It is then clear that the very-weak coupling regime will be inaccurate to describe emitter arrays with subwavelength separation distances. However, the coupling parameters decrease as the separation distance increases. Specifically, it can be concluded from the figure that for a configuration in which the separations are comparable to or larger than the wavelength of the transition frequency, the coupling can be safely neglected. We remark that the coupling in this and other configurations could be reduced 
further with the use of photonic structures (e.g., photonic band-gap materials [21,22] and metamaterials [23]).

\section{APPENDIX C: NONIDENTICAL EMITTERS}

In this appendix we generalize the theory introduced in Sec. II for the case of nonidentical emitters. In such a case, the electric field operator in the far zone will in general have two components, i.e., $\widehat{\mathbf{E}}^{(+)}(\mathbf{r}, t)=\sum_{a=\theta, \phi} \mathbf{u}_{a} \widehat{E}_{a}^{(+)}(\mathbf{r}, t)$, with

$$
\widehat{E}_{a}^{(+)}=\sum_{n=1}^{N} \frac{B_{n a}(\phi, \theta)}{r} \widehat{\sigma}_{n} e^{-\left(i \omega_{n}+\gamma_{n}\right) t_{d}} e^{-i \frac{\omega_{n}}{c} \mathbf{u}_{n} \cdot \mathbf{r}_{n}}
$$

and $B_{n a}(\phi, \theta)=-\mu_{0} \omega_{n}^{2} /(4 \pi) \mathbf{u}_{a} \cdot \mathbf{I}_{T} \cdot \mathbf{p}_{n}$. Similarly, the photon statistics must discriminate which polarization of the photons is being measured. For example, the probability density given by Eq. (3) is now generalized as

$$
\begin{aligned}
& P_{L}\left(\mathbf{u}_{1}, t_{1}, a_{1} ; \ldots ; \mathbf{u}_{L}, t_{L}, a_{L}\right) \\
& \propto r^{2 L}\left\langle\widehat{E}_{a_{1}, 1}^{(-)} \ldots \widehat{E}_{a_{L}, L}^{(-)} \widehat{E}_{a_{L}, L}^{(+)} \ldots \widehat{E}_{a_{1}, 1}^{(+)}\right\rangle .
\end{aligned}
$$

By introducing $(\mathrm{C} 1)$ into $(\mathrm{C} 2)$ we can calculate any directional, temporal, and polarization-based correlation. From a practical standpoint, it might be interesting to consider identical emitters with a variable polarization. In such a case, the time-integrated correlation (4) is generalized as follows:

$$
\begin{gathered}
g\left(\mathbf{u}_{1}, a_{1} ; \ldots ; \mathbf{u}_{L}, a_{L}\right) \propto A^{L} \sum_{n_{1}, \ldots, n_{L}} \sum_{m_{1}, \ldots, m_{L}} \prod_{l=1}^{L}\left|c_{a l}\left(\phi_{l}, \theta_{l}\right)\right|^{2} \\
\left\langle\widehat{\sigma}_{n_{1}}^{\dagger} \ldots \widehat{\sigma}_{n_{L}}^{\dagger} \widehat{\sigma}_{m_{L}} \ldots \widehat{\sigma}_{m_{1}}\right\rangle \prod_{l=1}^{L} e^{i \frac{\omega_{0}}{c} \mathbf{u}_{l} \cdot \mathbf{r}_{n_{l}}} e^{-i \frac{\omega_{0}}{c} \mathbf{u}_{l} \cdot \mathbf{r}_{m_{l}}}
\end{gathered}
$$

with $c_{a}(\phi, \theta)=\mathbf{u}_{a} \cdot \mathbf{I}_{T} \cdot \mathbf{u}_{n}$. This formalism is readily adapted to any initial state of the system. The final details will intimately depend on the practical properties of the emitters being considered for a specific application.

\section{APPENDIX D: MAXIMAL DIRECTIVITY IN THE AVERAGE NUMBER OF PHOTONS}

Here we demonstrate that quantum interference effects cannot increase the directivity of the average number of photons beyond the limits on directivity in the classical case. Our starting point is the first-order time integrated correlation function (average number of photons measured along the direction $\mathbf{u}_{1}$ )

$$
g_{1}\left(\mathbf{u}_{1}\right) \propto A \sin ^{2} \theta_{1} f_{1}\left(\mathbf{u}_{1}\right)
$$

with the first-order generalized array factor being given by

$$
f_{1}\left(\mathbf{u}_{1}\right)=\sum_{n, m=1}^{N}\left\langle\widehat{\sigma}_{n}^{\dagger} \widehat{\sigma}_{m}\right\rangle e^{i k_{0} \mathbf{u}_{1} \cdot \mathbf{r}_{n}} e^{-i k_{0} \mathbf{u}_{1} \cdot \mathbf{r}_{m}} .
$$

In the classical case we have $\left\langle\widehat{\sigma}_{n}^{\dagger} \widehat{\sigma}_{m}\right\rangle \rightarrow a_{n}^{*} a_{m}$, so that we can write

$$
f_{1}\left(\mathbf{u}_{1}\right)=\left|\sum_{m=1}^{N} a_{m} e^{-i k_{0} \mathbf{u}_{1} \cdot \mathbf{r}_{m}}\right|^{2} .
$$

Even if it were more general than the classical case, let us first analyze the constraints of $\left\langle\widehat{\sigma}_{n}^{\dagger} \widehat{\sigma}_{m}\right\rangle$ in the quantum case. To this end, we define a matrix $\mathbf{M}$ with elements $M_{n m}=\left\langle\widehat{\sigma}_{n}^{\dagger} \widehat{\sigma}_{m}\right\rangle$ and a vector $\mathbf{e}$ with elements $e_{n}=e^{-i k_{0} \mathbf{u}_{1} \cdot \mathbf{r}_{n}}$. This implies that $f_{1}\left(\mathbf{u}_{1}\right)$ can be written as

$$
f_{1}\left(\mathbf{u}_{1}\right)=\mathbf{e}^{\dagger} \cdot \mathbf{M} \cdot \mathbf{e} .
$$

First, we note that $\left\langle\widehat{\sigma}_{n}^{\dagger} \widehat{\sigma}_{m}\right\rangle=\left\langle\widehat{\sigma}_{m}^{\dagger} \widehat{\sigma}_{n}\right\rangle^{*}$ and, hence, $\mathbf{M}$ is a Hermitian matrix. In addition, since $f_{1}\left(\mathbf{u}_{1}\right)$ is a real positive number, we then have that $\mathbf{M}$ is a positive semidefinite matrix. Importantly, this implies that all its eigenvalues are positive. Next, let $\lambda_{j}$ and $\mathbf{m}_{j}$ be the eigenvalues and associated eigenvectors of $\mathbf{M}$. Then the spectral decomposition of $\mathbf{M}$ is given by

$$
\mathbf{M}=\sum_{j} \lambda_{j} \mathbf{m}_{j}^{\dagger} \cdot \mathbf{m}_{j} .
$$

Subsequently, $f_{1}\left(\mathbf{u}_{1}\right)$ can be rewritten as

$$
\begin{aligned}
f_{1}\left(\mathbf{u}_{1}\right) & =\sum_{j} \lambda_{j} \mathbf{e}^{\dagger} \cdot\left\{\mathbf{m}_{j}^{\dagger} \cdot \mathbf{m}_{j}\right\} \cdot \mathbf{e} \\
& =\sum_{j} \lambda_{j}\left|\sum_{n=1}^{N} m_{n(j)} e^{-i k_{0} \mathbf{u}_{1} \cdot \mathbf{r}_{n}}\right|^{2} .
\end{aligned}
$$

Equation (D6) demonstrates that the (quantum) generalized array factor describing the average number of photons measured as a function of direction can be written as a linear combination of classical array factors for the same array with different driving coefficients. Crucially, note that the $\lambda_{j}$ are always positive. Consequently, the contributions from each classical array factor are added in all directions. This decomposition suggests that the directivity cannot be enhanced, but only decreased, in the quantum case.

Specifically, the directivity can be written as

$$
\begin{aligned}
D_{1}\left(\mathbf{u}_{1}\right) & =4 \pi \frac{g_{1}\left(\mathbf{u}_{1}\right)}{\int d \Omega_{1} g_{1}\left(\mathbf{u}_{1}\right)} \\
& =4 \pi \frac{\sin ^{2} \theta_{1} \sum_{j} \lambda_{j}\left|\sum_{n=1}^{N} m_{n(j)} e^{-i k_{0} \mathbf{u}_{1} \cdot \mathbf{r}_{n}}\right|^{2}}{\sum_{j} \lambda_{j} \int d \Omega_{1} \sin ^{2} \theta_{1}\left|\sum_{n=1}^{N} m_{n(j)} e^{-i k_{0} \mathbf{u}_{1} \cdot \mathbf{r}_{n}}\right|^{2}} .
\end{aligned}
$$

This situation is identical that obtained in the classical case for orthogonal polarization channels. The latter cannot increase the directivity since those channels contribute independently to the power carried into the chosen direction and the integral over all directions. This can be proven by noting that if we optimize the $m_{n(j)}$ coefficients, we would obtain the same optimal coefficients for any $j$, and therefore the sums in the numerator and denominator would compensate in obtaining the maximal directivity. Consequently, the directivity of a quantum emitter array cannot exceed that of the corresponding classical antenna array.

Aside from this theoretical result, the decomposition (D6) also brings some insight into the directivity effects of some specific examples. For example, let us consider the decay from $\mathrm{a}\left|\psi_{S N}\right\rangle=\prod_{n=1}^{N} \widehat{\sigma}_{n}^{\dagger}|0\rangle$ state, for which we obtain $\left\langle\widehat{\sigma}_{n}^{\dagger} \widehat{\sigma}_{m}\right\rangle=$ $\delta_{n m}$. This means that the matrix $\mathbf{M}$ is a diagonal matrix. This matrix can be decomposed into a basis of eigenvectors: $(1,0, \ldots, 0),(0,1,0, \ldots, 0)$, and so on. In turn, this means that $f_{1}\left(\mathbf{u}_{1}\right)$ can be constructed as the individual addition of the array factor of each individual emitter and, hence, $f_{1}\left(\mathbf{u}_{1}\right)=N$. 
[1] R. H. Dicke, Phys. Rev. 93, 99 (1954).

[2] M. Gross and S. Haroche, Phys. Rep. 93, 301 (1982).

[3] A. Asenjo-Garcia, M. Moreno-Cardoner, A. Albrecht, H. J. Kimble, and D. E. Chang, Phys. Rev. X 7, 031024 (2017).

[4] M. O. Scully, Phys. Rev. Lett. 102, 143601 (2009).

[5] R. Röhlsberger, K. Schlage, B. Sahoo, S. Couet, and R. Rüffer, Science 328, 1248 (2010).

[6] A. Delga, J. Feist, J. Bravo-Abad, and F. J. Garcia-Vidal, Phys. Rev. Lett. 112, 253601 (2014).

[7] R. Sáez-Blázquez, J. Feist, A. I. Fernández-Domínguez, and F. J. García-Vidal, Optica 4, 1363 (2017).

[8] C. Noh and D. G. Angelakis, Rep. Prog. Phys. 80, 016401 (2017).

[9] K. Hammerer, A. S. Sørensen, and E. S. Polzik, Rev. Mod. Phys. 82, 1041 (2010).

[10] H. Bernien, S. Schwartz, A. Keesling, H. Levine, A. Omran, H. Pichler, S. Choi, A. S. Zibrov, M. Endres, M. Greiner et al., Nature (London) 551, 579 (2017).

[11] J. Zhang, G. Pagano, P. W. Hess, A. Kyprianidis, P. Becker, H. Kaplan, A. V. Gorshkov, Z.-X. Gong, and C. Monroe, Nature (London) 551, 601 (2017).

[12] M. Endres, H. Bernien, A. Keesling, H. Levine, E. R. Anschuetz, A. Krajenbrink, C. Senko, V. Vuletic, M. Greiner, and M. D. Lukin, Science 354, 1024 (2016).

[13] H. Labuhn, D. Barredo, S. Ravets, S. De Léséleuc, T. Macrì, T. Lahaye, and A. Browaeys, Nature (London) 534, 667 (2016).

[14] J. L. Zhang, K. G. Lagoudakis, Y.-K. Tzeng, C. Dory, M. Radulaski, Y. Kelaita, K. A. Fischer, S. Sun, Z.-X. Shen, N. A. Melosh, S. Chu, and J. Vučković, Optica 4, 1317 (2017).

[15] C. Palacios-Berraquero, D. M. Kara, A. R.-P. Montblanch, M. Barbone, P. Latawiec, D. Yoon, A. K. Ott, M. Loncar, A. C. Ferrari, and A. Mete, Nat. Commun. 8, 15093 (2017).

[16] C. A. Balanis, Antenna Theory: Analysis and Design (John Wiley \& Sons, New York, 2005).

[17] R. C. Hansen, Phased Array Antennas, 2nd ed. (John Wiley \& Sons, New York, 2009).

[18] R. J. Mailloux, Phased Array Antenna Handbook, Vol. 2 (Artech House, Boston, 2005).
[19] P.-S. Kildal, Foundations of Antenna Engineering: A Unified Approach for Line-of-Sight and Multipath (Artech House, Boston, 2015).

[20] J. S. Herd and M. D. Conway, Proc. IEEE 104, 519 (2016).

[21] F. Yang and Y. Rahmat-Samii, IEEE Trans. Antennas Propag. 51, 2936 (2003).

[22] R. Gonzalo, P. D. Maagt, and M. Sorolla, IEEE Trans. Microw. Theory Techn. 47, 2131 (1999).

[23] M.-C. Tang, Z. Chen, H. Wang, M. Li, B. Luo, J. Wang, Z. Shi, and R. W. Ziolkowski, IEEE Trans. Antennas Propag. 65, 3986 (2017).

[24] H. Steyskal and J. S. Herd, IEEE Trans. Antennas Propag. 38, 1971 (1990).

[25] R. Loudon, The Quantum Theory of Light (Oxford University Press, Oxford, 2000).

[26] M. O. Scully and M. S. Zubairy, Quantum Optics (Cambridge University Press, Cambridge, 1997).

[27] S. M. Barnett and P. M. Radmore, Methods in Theoretical Quantum Optics (Oxford University Press, Oxford, 2002).

[28] R. J. Glauber, Phys. Rev. Lett. 10, 84 (1963).

[29] R. J. Glauber, Phys. Rev. 130, 2529 (1963).

[30] P. Kelley and W. Kleiner, Phys. Rev. 136, A316 (1964).

[31] H. J. Carmichael, Statistical Methods in Quantum Optics 2: Non-Classical Fields (Springer Science \& Business Media, New York, 2009).

[32] A. Predojević and M. W. Mitchell, Engineering the Atom-Photon Interaction (Springer, New York, 2015).

[33] M. Saffman, T. G. Walker, and K. Mølmer, Rev. Mod. Phys. 82, 2313 (2010).

[34] A. Grankin, D. Vasilyev, P. Guimond, B. Vermersch, and P. Zoller, arXiv:1802.05592 (2018).

[35] W. Vogel and D.-G. Welsch, Quantum Optics (John Wiley \& Sons, New York, 2006).

[36] H. T. Dung, L. Knöll, and D.-G. Welsch, Phys. Rev. A 66, 063810 (2002).

[37] H.-P. Breuer and F. Petruccione, The Theory of Open Quantum Systems (Oxford University Press on Demand, Oxford, 2002).

[38] I. Liberal and N. Engheta, Phys. Rev. A 97, 022309 (2018). 